\title{
MINI ROBÔ AUTÔNOMO PARA COMPETIÇÃO DE SUMÔ
}

\section{Andre Luiz Silva de Oliveira ${ }^{1}$ \\ João Paulo Teixeira ${ }^{2}$ \\ Juliano Sansão ${ }^{3}$ \\ Rafael Hashinaga ${ }^{4}$ \\ Rafael Cardoso ${ }^{5}$ \\ Jonatas Alves ${ }^{6}$ \\ Helosman Valente ${ }^{7}$}

Resumo: Este trabalho descreve o projeto de construção do sumô de robô, que tem como principal objetivo produzir conhecimento da prática acerca de robótica, automação, programação, comandos elétricos, desenvolvimento de peças. O sumô de robô consiste em uma modalidade de competição de lutas contra robôs, que assemelha ao sumô humano. A elaboração e construção deste robô foram necessários à utilização de microcontroladores, sensores, protocolos de comunicação, sistema de tração e integração de sistemas de diferentes disciplinas, como mecânica, elétrica e controle. O robô de sumô é um passo importante no processo do desenvolvimento de conhecimento na área de automação, pois qualquer robô autônomo necessita da integração entre mecânica, eletrônica e software.

Palavras-chave: Sumô de robô; Programação; Microcontroladores; Sensores.

\footnotetext{
${ }^{1}$ Engenharia Elétrica/Universidade do Vale do Paraíba, Brasil. E-mail: aluizsoliveira@gmail.com.

2 Engenharia Elétrica/Universidade do Vale do Paraíba, Brasil. E-mail: joao_p.t@hotmail.com.

${ }^{3}$ Engenharia Elétrica/Universidade do Vale do Paraíba, Brasil. E-mail: juliano.sansao@electricdreams.com.br.

${ }^{4}$ Engenharia Elétrica/Universidade do Vale do Paraíba, Brasil. E-mail: rafaelhashinaga@gmail.com.

${ }^{5}$ Engenharia Elétrica/Universidade do Vale do Paraíba, Brasil. E-mail: cardoso95rafael@outlook.com.

${ }^{6}$ Engenharia Elétrica/Universidade do Vale do Paraíba, Brasil. E-mail: jonatasalves.r@gmail.com.

${ }^{7}$ Engenharia Elétrica/Universidade do Vale do Paraíba, Brasil. E-mail: helosman@gmail.com.
} 